\title{
BMJ Open Knee Arthroscopy Cohort Southern Denmark (KACS): protocol for a prospective cohort study
}

\author{
Jonas Bloch Thorlund, ${ }^{1}$ Robin Christensen, ${ }^{1,2}$ Nis Nissen, ${ }^{3}$ Uffe Jørgensen, ${ }^{4}$ \\ Jeppe Schjerning, ${ }^{5}$ Jens Christian Pørneki, ${ }^{6}$ Martin Englund, ${ }^{7,8}$ \\ L Stefan Lohmander $^{1,4,7}$
}

To cite: Thorlund JB, Christensen R, Nissen N, et al. Knee Arthroscopy Cohort Southern Denmark (KACS): protocol for a prospective cohort study. BMJ Open 2013;3:e003399. doi:10.1136/bmjopen-2013003399

- Prepublication history and additional material for this paper is available online. To view these files please visit the journal online (http://dx.doi.org/10.1136/ bmjopen-2013-003399).

Received 13 June 2013 Revised 22 August 2013 Accepted 30 August 2013

For numbered affiliations see end of article.

Correspondence to Dr Jonas Bloch Thorlund; jthorlund@health.sdu.dk

\section{ABSTRACT}

Background: Meniscus surgery is a high-volume surgery carried out on 1 million patients annually in the USA. The procedure is conducted on an outpatient basis and the patients leave the hospital a few hours after surgery. A critical oversight of previous studies is their failure to account for the type of meniscal tears. Meniscus tears can be categorised as traumatic or nontraumatic. Traumatic tears (TT) are usually observed in younger, more active individuals in an otherwise 'healthy' meniscus and joint. Non-traumatic tears (NTT) (ie, degenerative tears) are typically observed in the middleaged (35-55 years) and older population but the aetiology is largely unclear. Knowledge about the potential difference of the effect of arthroscopic meniscus surgery on patient symptoms between patients with traumatic and NTT is sparse. Furthermore, little is known about the natural time course of patient perceived pain, function and quality of life after meniscus surgery and factors affecting these outcomes. The aim of this prospective cohort study is to investigate the natural time course of patient-reported outcomes in patients undergoing meniscus surgery, with particular emphasis on the role of type of symptom onset.

Methods/design: This prospective cohort study enrol patients assigned for meniscus surgery. At the baseline (PRE surgery), patient characteristics are assessed using an email-based questionnaire also comprising several validated questionnaires assessing general health, knee-specific characteristics and patient's expectations of the surgery. Follow-up will be conducted at 12 and 52 weeks after meniscus surgery. The major outcomes will be differences in changes, from before to 52 weeks after surgery, in each of the five domains on the Knee injury and Osteoarthritis Outcome Score (KOOS) between patients undergoing surgery for traumatic compared with non-traumatic meniscus tears.

Dissemination: The study findings will be disseminated in peer-reviewed journals and presented at national and international conferences.

Trial registration number: ClinicalTrials.gov Identifier: NCT01871272.

\section{INTRODUCTION}

Meniscus surgery is a high-volume surgery carried out on 1 million patients annually in

\section{ARTICLE SUMMARY}

Strengths and limitations of this study

- This cohort study collects data on the natural time course of patient reported outcomes in a clinical setting on a large group of patients after arthroscopic meniscus surgery to ensure high external validity.

- As data is collected on a large number of patients in a clinical setting it was not feasible to collect standardised imaging data (ie, MRI or radiographs) on patients, which could have provided valuable information.

the USA. ${ }^{1}$ The procedure is conducted on an outpatient basis and patients leave the hospital few hours after surgery. Nevertheless, little is known about the natural time course of patient perceived pain, function and quality of life (QOL) after meniscus surgery and which factors affect these outcomes. ${ }^{2}$ The general opinion is that patients recover their muscle strength fully within 6-12 weeks following arthroscopic partial meniscectomy. ${ }^{3-5}$ More importantly, however, recent studies have shown substantial patient-reported disability and pain in patients up to 4 years after surgery. ${ }^{6-8}$ One explanation for the poor selfreported outcomes may be that the loss of meniscal function triggers other events that may cause knee pain. ${ }^{9}$ Complicating the assessment of surgery effectiveness further, surgical procedures have shown to be associated with considerable 'placebo effect'. 1011

A critical limitation of previous studies ${ }^{12-15}$ is their failure to account for the type of symptom onset (ie, injury mechanism). Meniscus tears can be categorised as either traumatic or non-traumatic. Traumatic tears (TT) are usually observed in younger, active individuals in an otherwise 'healthy' meniscus and joint, and can be attributed to a specific incident (eg, sports-related trauma) ${ }^{16}$ TT's are often associated with joint effusion, 
reduced knee joint range of motion (ROM) together with catching/locking of the knee. Non-traumatic tears (NTT) are typically observed in the middle-aged ( $35-55$ years) and older population. ${ }^{17}$ These tears are associated with meniscal calcification ${ }^{18}$ and risk factors for these tears include, presence of Heberdens's and Bouchard nodes, knee malalignment ${ }^{19}$ and occupational kneeling ${ }^{20}$; however, the aetiology is largely unclear. ${ }^{16}$ NTT's are often referred to as degenerative tears and have been shown to be associated with incipient knee osteoarthritis (OA) in the middle-aged or elderly population. ${ }^{21-23}$ Evidence from four well-designed trials demonstrated that arthroscopic interventions ${ }^{1024}$ and meniscectomy ${ }^{25-27}$ were no better or provided no additional effect, than the comparator (ie, sham surgery, physical therapy or a combination of physical and medical therapy) to relieve pain and improve function in the middle-aged patients with knee $\mathrm{OA}$ or early signs of knee OA. No corresponding randomised trials exist specifically for TT but an observational study showed that patients with degenerative meniscus lesions (ie, NTT) self-report worse function and QOL compared to individuals with TT at follow-up 14 years after meniscectomy. ${ }^{28}$ Thus, it is conceivable, but currently unproven, that arthroscopic meniscus surgery is more effective in resolving symptoms of a meniscus tear of traumatic aetiology compared with non-NTT in the middle-aged population.

In patients with TT, repair of the meniscus may be an alternative to resection. In contrast, repair is rarely an option for middle-aged patients with NTT due to the degenerative state of the meniscus. A recent retrospective observational study suggested a reduced risk of later knee OA and less activity level loss in patients ( $~ 32$ years at time of surgery) undergoing repair compared with resection (ie, favouring repair). ${ }^{29}$ This indicates that patients with TT should be stratified into subgroups on the basis of type of arthroscopic intervention (ie, repair $\left(\mathrm{TT}_{\mathrm{REP}}\right)$ and resection $\left.\left(\mathrm{TT}_{\mathrm{RES}}\right)\right)$ since this may influence the patient-perceived outcomes after surgery.

\section{Aims and hypotheses}

The primary aims of this observational cohort are to

1. Investigate if improvements in patient self-reported pain, symptoms, function and QOL differ after arthroscopic meniscus surgery for non-traumatic meniscus tears in middle-aged patients, compared with surgery in patients with traumatic tears (ie, NTT vs TT). We hypothesise that in middle-aged patients with NTT arthroscopic surgery is less effective in relieving self-reported pain, symptoms, function in sports and recreation (Sport/ Rec) and QOL (ie, change in KOOS scores), compared with younger patients undergoing surgery for TT.

2. Investigate the effect of meniscus repair $\left(\mathrm{TT}_{\mathrm{REP}}\right)$ compared to meniscus resection $\left(\mathrm{TT}_{\mathrm{RES}}\right)$ on change in self-reported pain, symptoms, function in Sport/ Rec and QOL in patients with TT. We hypothesise that arthroscopic surgery is less effective in relieving pain, symptoms, function in Sport/Rec and QOL (ie, change in KOOS scores) in patients undergoing $\mathrm{TT}_{\mathrm{RES}}$ compared with those undergoing $\mathrm{TT}_{\mathrm{REP}}$

\section{METHODS AND ANALYSIS}

\section{Design}

In this prospective cohort study we will assess patientreported outcomes (PROs) using email-based questionnaires prior to surgery and at 12 and 52 weeks follow-up postsurgery (see figure 1).

\section{Participants}

All patients assigned for arthroscopy on suspicion of a meniscus tear at Lillebælt Hospital (located in the cities Vejle and Kolding, Denmark) and Odense University Hospital, Denmark (incl. Svendborg Hospital) from 1 February 2013 to 31 January 2014.

\section{General cohort eligibility criteria}

Inclusion criteria: patients $\geq 18$ years of age assigned for arthroscopy on suspicion of a medial and/or lateral meniscus tear by the examining orthopaedic surgeon based on clinical signs and MRI (if available), having an email address and able to read and understand Danish.

Exclusion criteria: patients who will or previously have undergone surgical reconstruction of the anterior or posterior cruciate ligament (ACL or PCL) in either knee, experienced fracture(s) to the lower extremities (ie, hip, leg or foot) in either leg within the last 6 months at time of recruitment and patients not
Figure 1 Overview of collection of outcomes during the first year in the Knee Arthroscopy Cohort Southern Denmark.

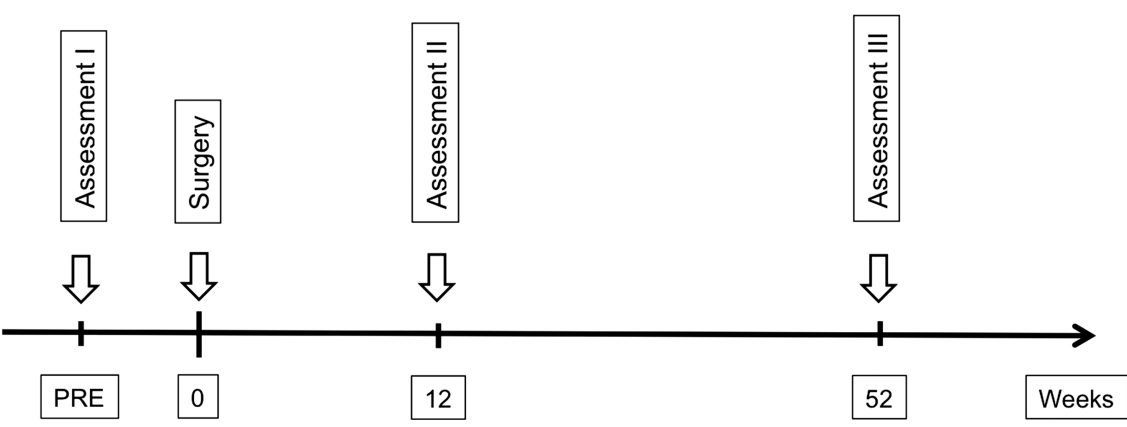


mentally able to reply the questionnaire. Please refer to figure 2 for an overview of the recruitment flow.

The patients with reconstructed ACL and PCL cannot be included as these patients are being followed in another cohort study.

\section{Inclusion and exclusion criteria, aim 1 (NTT vs TT)}

There is no consensus on how to classify patients as having a NTT or TT. In this study, patients undergoing meniscus surgery will be classified as having either TT or NTT according to an algorithm based on age, duration of knee symptoms and a question about injury mechanism (see below). This represents the information that is available prior to surgery.

\section{Injury mechanism question}

'How did the knee pain/problems for which you are now having surgery develop (choose the answer that best match your situation)?'

\section{Response alternatives}

A. The pain/problems have slowly evolved over time.

B. As a result of a specific incident (ie, kneeling, sliding and/or twisting of the knee or the like).
C. As a result of a violent incident (ie, during sports, a crash, collision or the like).

\section{TT}

Inclusion: all patients between 18 and 34 years and all patients between 35 and 55 years replying ' $\mathrm{C}$ ' on the injury mechanism question.

\section{NTT}

Inclusion: all patients between 35 and 55 years replying ' $\mathrm{A}$ ' or ' $\mathrm{B}$ ' on the injury mechanism question and having knee symptoms $>6$ months.

In addition, the general eligibility criteria also apply. For aim 1, the upper age limit is set to include patients with degenerative meniscus tears (ie, NTT) but without severe features of knee OA. ${ }^{30}$ Furthermore, patients whose responses do not fit the TT and NTT criteria will also be excluded.

\section{Inclusion and exclusion criteria, aim 2 ( $\mathrm{TT}_{\text {RES }}$ vs $\mathrm{TT}_{\mathrm{REP}}$ )}

All patients classified as TT according to the specific Knee Arthroscopy Cohort Southern Denmark (KACS) eligibility criteria for aim 1 will be further divided in patients having either meniscus resection $\left(\mathrm{TT}_{\mathrm{RES}}\right)$ or
Figure 2 Overview of the recruitment flow in the Knee Arthroscopy Cohort Southern Denmark.

\section{Recruitment flow}

All patients conforming with inclusion criteria are invited to participate in the study when assigned for meniscus surgery

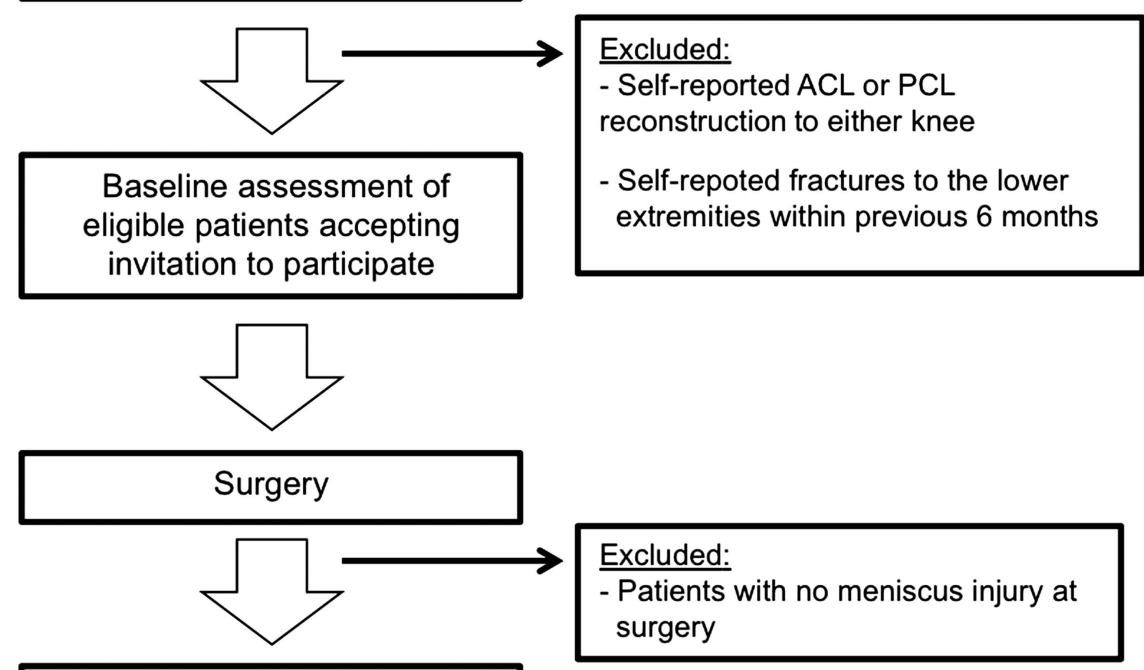

Patients eligible for 12 weeks follow-up assessment

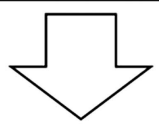

Patients eligible for 52 weeks follow-up assessment 
repair $\left(\mathrm{TT}_{\mathrm{REP}}\right)$ according to the type of surgery they receive to answer study aim 2.

\section{Patient characteristics}

At baseline, self-report information about: educational level, employment, civil status, smoking habits, comorbidities, ${ }^{31}$ physical activity level ${ }^{32}$ and self-reported knee and foot alignment ${ }^{33}$ will be collected together with information on height and weight. Surgery documentation will be collected using a modified version of the International Society of Arthroscopy, Knee Surgery and Orthopaedic Sports Medicine (ISAKOS) classification of meniscal tears questionnaire,${ }^{34}$ which is filled out by the operating surgeon. Additional surgery information not pertaining to the meniscus is also collected from surgery reports.

\section{Major outcomes}

Knee injury and Osteoarthritis Outcome Score (KOOS)

All 5 domains (ie, subscales) on the $\operatorname{KOOS}^{35} 36$ at the 1-year follow-up. The five KOOS domains are pain, symptoms, function during daily activities (ADL), Sport/ Rec function and QOL. The KOOS score is ranging from 0 to 100 (0 indicating extreme symptoms and 100 indicating no symptoms). The KOOS score has been validated and previously used to assess self-reported outcomes in patients undergoing meniscus surgery. ${ }^{6} 825273536$ In addition, it has been shown to perform well in the entire continuum from very early changes of knee OA to knee arthroplasty. ${ }^{37}$ All outcomes included in the study are listed in table 1.

\section{Minor outcomes}

\section{Patient Acceptable Symptom State (PASS) and Treatment} Failure (TF)

One question regarding PASS will be used to assess how many patients consider themselves well after surgery (as opposed to feeling better) ${ }^{38}$ PASS is assessed as a dichotomous outcome $(\mathrm{y} / \mathrm{n})$ to the question: "Considering your knee function, do you feel that your current state is satisfactory? With knee function you should take into account all activities you have during your daily life, Sport/Rec activities, your level of pain and other symptoms, and also your knee related QOL".

In addition, patients replying 'no' to the PASS question will also be asked to answer $(\mathrm{y} / \mathrm{n})$ the following question: "Would you consider your current state as being so unsatisfactory that you think the treatment has failed?" Patients replying, 'yes' to the second question will be defined as experiencing 'treatment failure' (TF).

\section{Medical outcomes study 36-item short form health survey (SF-36)}

The SF-36 will be used to assess general physical function. The SF-36 consists of eight subscales: physical function, role physical, bodily pain, general health, vitality, social function, role emotional and mental health. The SF-36 is self-explanatory, takes $10 \mathrm{~min}$ to complete and is
Table 1 Collection of patient characteristics, outcome measures and explanatory variables

\begin{tabular}{|c|c|c|c|c|}
\hline Variable & PRE & Surgery & 12 Weeks & 52 Weeks \\
\hline Height & $x$ & & & \\
\hline Weight & $x$ & & $x$ & $x$ \\
\hline Civil status & $x$ & & & \\
\hline $\begin{array}{l}\text { Educational } \\
\text { level }\end{array}$ & $x$ & & & \\
\hline Employment & $x$ & & & \\
\hline Smoking & $x$ & & & \\
\hline Comorbidities & $x$ & & & \\
\hline Alignment & $x$ & & & $x$ \\
\hline $\begin{array}{l}\text { Physical activity } \\
\text { level }\end{array}$ & $x$ & & & \\
\hline $\begin{array}{l}\text { ISAKOS } \\
\text { questionnaire }\end{array}$ & & $x$ & & \\
\hline $\begin{array}{l}\text { Knee joint } \\
\text { stability }\end{array}$ & $x$ & & $x$ & $x$ \\
\hline $\begin{array}{l}\text { Expectations for } \\
\text { surgery }\end{array}$ & $x$ & & & \\
\hline SF-36 & $x$ & & $x$ & $x$ \\
\hline KOOS & $x$ & & $x$ & $x$ \\
\hline PASS & & & $x$ & $x$ \\
\hline $\mathrm{TF}$ & & & $x$ & $x$ \\
\hline GPE & & & $x$ & $x$ \\
\hline$A E$ & & & $x$ & $x$ \\
\hline
\end{tabular}

$\mathrm{AE}$, adverse events; GPE, global perceived effect; ISAKOS, International Society of Arthroscopy, Knee Surgery and Orthopaedic Sports Medicine-classification of meniscal tears questionnaire; KOOS, knee injury and osteoarthritis outcome score; PASS, patient acceptable symptom state; SF-36, medical outcomes study 36-Item Short Form Health Survey;

$\mathrm{TF}$, treatment failure.

scored from 0 to 100 (0 indicating extreme problems and 100 indicating no problems). The Acute Danish version of the SF-36 was used. ${ }^{39} 40$

\section{Exploratory outcomes}

- Questions regarding patient's expectations of surgery. ${ }^{41}$

- Questions concerning knee joint stability/laxity. One question regarding the frequency of symptoms and one question about the influence of symptoms (ie, sense of instability during daily activities).

- Questions regarding postoperative rehabilitation (ie, participation, type, frequency and degree of supervision).

- Questions regarding global perceived effect (GPE) to explore minimal clinical important change in PROs. GPE is evaluated on a seven-step global rating scale after surgery (ranging from better, an important improvement; somewhat better, but enough to be an important improvement; very small change, not enough to be an important improvement; about the same; very small change, not enough to be an important worsening; somewhat worse, but enough to be an important worsening; worse, an important worsening). A two-step change in GPE is considered clinically important. ${ }^{42}$ 


\section{Adverse events}

Adverse events (not necessarily implying causality to the surgery), defined as self-reported symptoms after surgery causing limitations in daily activities, Sport/Rec activities or work limitations together with symptoms causing patients to seek medical care or having re-surgery will be collected by self-report and patient record review.

\section{Data management}

All self-reported data are collected using email-based questionnaires. The participant-submitted responses are automatically registered in a secured database. At all data collection points an email reminder is sent to participants if they do not answer the email-based questionnaire within 3-4 days. In addition, participants who do not reply after the reminder will be called by phone to ensure a high follow-up rate.

Information registered by surgeons on the modified ISAKOS questionnaire following surgery will be transferred from paper format to electronic format using automated forms processing. This method is a validated alternative to double entry of data. ${ }^{43}$

\section{Statistical analysis}

The cohort will recruit all eligible patients from 1 February 2013 to 31 January 2014. Conservatively estimated we expect to recruit 450 patients to the KACS cohort within this time frame. For an overview of the expected distribution of patients recruited between 18 and 55 years, please refer to figure 3 .

The minimal clinically important change on the KOOS subscale is considered to be $8-10$ points. ${ }^{37}$ Thus, with the estimated recruitment flow and distribution (figure 3); we will have a power of 0.99 for a two-sample pooled $\mathrm{t}$ test of a normal mean difference with a twosided significance level of $0.05(\mathrm{p} \leq 0.05)$, assuming a common SD of 15 KOOS points to detect a mean difference of 8 KOOS points between NTT and TT (primary study aim 1).

In addition, we will have a power of 0.88 for a twosample pooled t test of a normal mean difference with a two-sided significance level of 0.05 ( $p \leq 0.05)$, assuming a common SD of 15 KOOS points to detect a mean difference of 10 KOOS points between $\mathrm{TT}_{\mathrm{RES}}$ and $\mathrm{TT}_{\mathrm{REP}}$ (study aim 2).

If we are not able to reach sufficient numbers within the 1 year timeframe, recruitment will continue until the numbers specified in the a priori sample size calculation are reached.

Descriptive results will be given as means with SDs (or medians with IQR) and as percentages. Between-group comparisons of the KOOS and SF-36 scores at the 52 weeks of follow-up will be analysed with the use of ANalysis of COVAriance (ANCOVA), stratified by site and adjusted for the preoperative score level, sex, age and body mass index (BMI). PASS and TF will be analysed using $\chi^{2}$ test. Multiple logistic regression will be applied to estimate ORs for dichotomous outcomes. Mixed linear effects models with patient as random factor and sex, age and BMI as fixed factors will be used to explore change over time (ie, baseline, 12 weeks and 52 weeks of follow-up) in KOOS and SF-36 scores. Results will be presented with 95\% CIs. No interim analysis will be performed. All reported $p$ values are two-sided and will not be adjusted for multiple comparisons. All data analyses will be carried out according to the pre-established analysis plan. All descriptive statistics and tests will be reported in accordance to the recommendations of the 'Enhancing the QUAlity and Transparency Of health Research' (EQUATOR) network: the STROBE statement. ${ }^{44}$

\section{Full analysis set}

To qualify for the 'full analysis set' recruited patients must reply to the baseline questionnaire and have the surgery performed to their meniscus. Please refer to figure 4, for an overview of the full analysis set. In case of missing data a non-responder imputation will be applied (ie, baseline observation carried forward). Further for sensitivity, the effect that any missing data might have on results will be assessed through sensitivity analyses of augmented data sets.

\section{Planned sensitivity analysis}

Sensitivity analysis will be conducted to explore whether the degree of cartilage defects (score: $0-4$ ), and plica presence $(y / n)$ have any impact on the outcome after surgery. Furthermore, we will construct a dichotomous outcome on whole knee OA $(\mathrm{y} / \mathrm{n})$ to explore the effect of presence of knee OA. Whole knee OA will be defined as; participants with cartilage defects International Cartilage Repair Society grade $>2$ in either of the patellofemoral, medial tibiofemoral or lateral tibiofemoral compartment excluding participants with TT (according to previous definition) and symptoms $<6$ months. In
Figure 3 Expected distribution per 450 patients recruited, divided by age, type of tear and type of surgery. NTT, non-traumatic tear; $\mathrm{TT}$, traumatic tear; $\mathrm{TT}_{\mathrm{RES}}$, traumatic tear resected; $\mathrm{TT}_{\mathrm{REP}}$, traumatic tear repaired.
Expected distribution per 450 recruited patients Age $\quad$ Type of tear $\quad$ Type of surgery

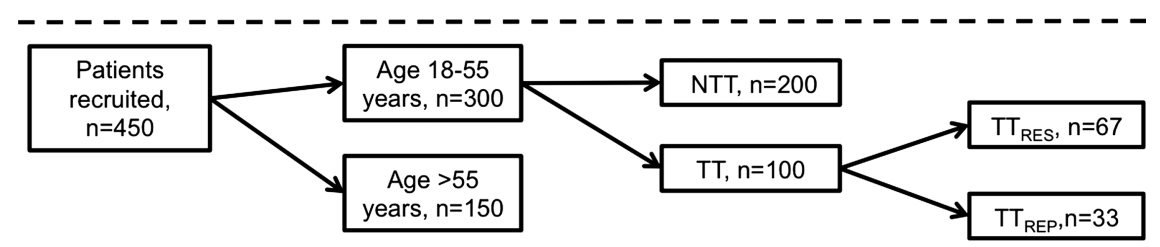




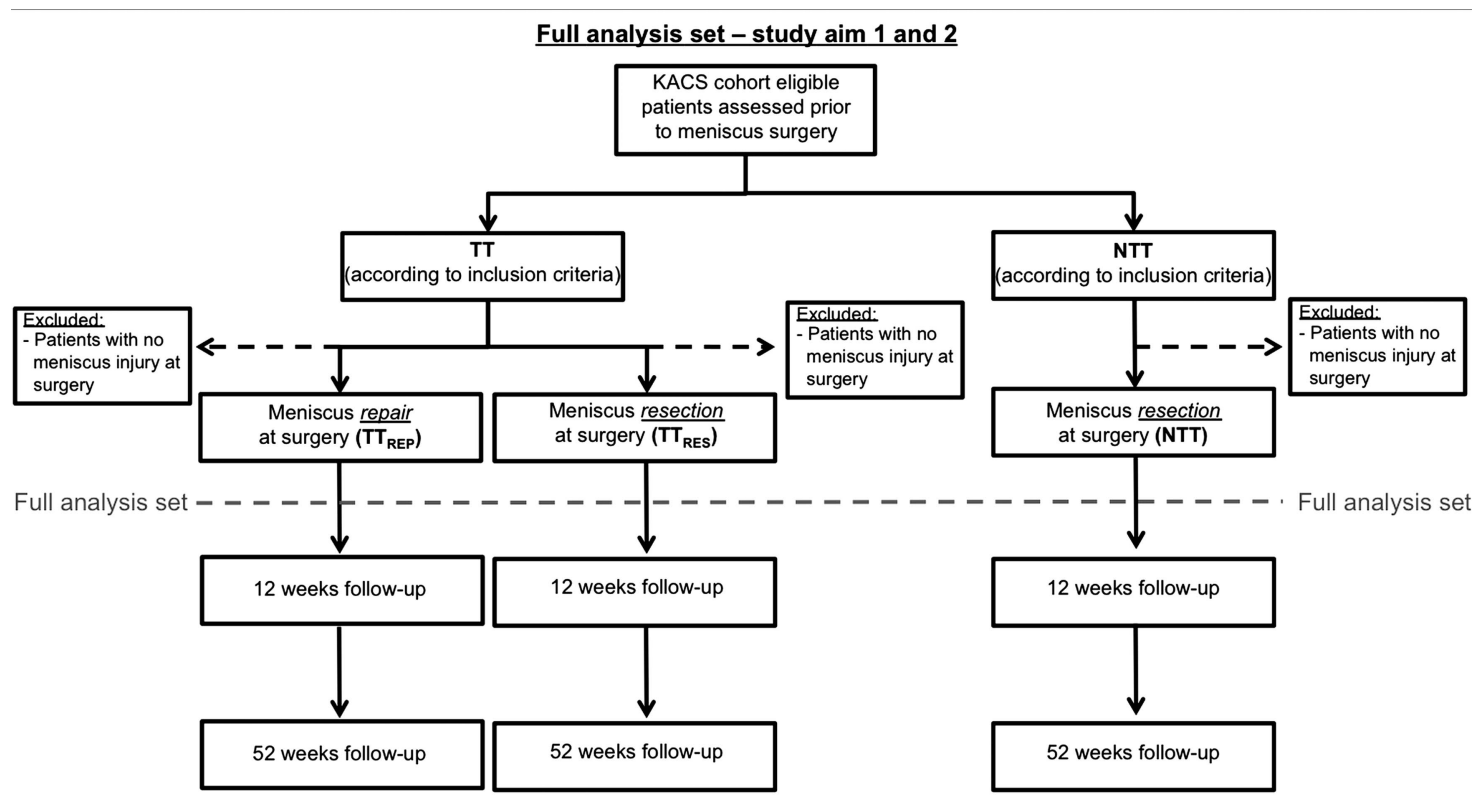

Figure 4 Overview of the full-analysis set for study aims 1 and 2. NTT, non-traumatic tear; $\mathrm{TT}$, traumatic tear; $\mathrm{TT}_{\mathrm{RES}}$, traumatic tear resected; $\mathrm{TT}_{\mathrm{REP}}$, traumatic tear repaired.

addition, the effect of differences in patient characteristics between groups reported in table 1 with a $\mathrm{p}$ value $\leq 0.10$ will be tested in a fully adjusted model.

\section{ETHICS AND DISSEMINATION}

The Regional Scientific Ethics Committee of Southern Denmark has reviewed the outline of this cohort study. The committee waived the need for ethical approval as study is the only pertaining questionnaire and register data. Such studies can be implemented without permission from the Ethics Committee according to Danish legislation (Committee Act $\S 1$, paragraph 1).

The study findings will be disseminated in peerreviewed journals and presented at national and international conferences.

\section{DISCUSSION}

Arthroscopic meniscus surgery is a high-volume surgery. ${ }^{1}$ Little is known about the natural time course of PROs after meniscus surgery and which factors affect these outcomes. This prospective cohort will collect data from a large number of patients on the natural time course of PROs prior and following arthroscopic meniscus surgery. Our results will enable analysis of the dependence of postsurgery outcome on the type of meniscus tear (ie, TT vs NTT in middle-aged patients). Further, it will be possible to investigate the dependence of postsurgery outcome on the type of surgery in the subgroup of patients with $\mathrm{TT}$ (ie, $\mathrm{TT}_{\mathrm{RES}}$ vs $\mathrm{TT}_{\mathrm{REP}}$ ). In contrast, other on-going randomised placebo controlled trials are investigating the effect of meniscus surgery for patients with degenerative tears. ${ }^{45} 46$
In this study a pragmatic clinical approach was chosen to categorise meniscus tears as either TT or NTT (ie, degenerative). The advantages of this approach are that it is simple, cheap, can be determined prior to surgery (in contrast to histology or arthroscopic observation) and feasible in a routine clinical setting. Thus, this information can be used to form an algorithm based on information available prior to surgery to select those patients who benefit most from surgery, which can be implemented in clinical practice. The definition of TT and NTT are similar but not identical to what has previously been used in other studies. Camanho et $a l^{12}$ divided the patients into three groups; traumatic, degenerative and fatigue. In the present study the NTT group will include degenerative as well as fatigue as defined by Camanho et al as the focus of this study is on the traumatic versus non-traumatic initiation of the meniscal tear. Others have based their definition on sports participation. ${ }^{47}$

A limitation to this study is that patients are included based on the main reason for surgery (ie, suspicion of a meniscus tear). However, meniscus surgery may also be performed in relation to surgery for other knee pathologies. Those patients will not be included in the KACS. This should be taken into account when interpreting the cohort data. On the other hand, this makes it more likely that patient symptoms in the KACS cohort are primarily caused by the meniscus injury. Furthermore, we expect the age to be different in the TT compared with the NTT groups (ie, NTT group being older), thus all statistical analysis will be adjusted for age. Nevertheless, this should still be taken into consideration when interpreting the results.

Meniscus surgery may not be the answer to improve patient-perceived pain and function in all patients with 
meniscus tears. Different factors, such as type of tear, may affect the postoperative outcome. Ultimately the goal of this study is to improve management of patients with meniscus tears through identifying factors associated with no or limited effect of surgery on PROs.

\section{Author affiliations}

${ }^{1}$ Department of Sports Science and Clinical Biomechanics, University of Southern Denmark, Odense, Denmark

${ }^{2}$ Musculoskeletal Statistics Unit, The Parker Institute, Depart of Rheumatology, Copenhagen University Hospital, Frederiksberg, Copenhagen F, Denmark ${ }^{3}$ Department of Orthopedics, Lillebaelt Hospital, Kolding, Denmark ${ }^{4}$ Department of Orthopedics and Traumatology, Odense University Hospital, Odense, Denmark

${ }^{5}$ Department of Orthopedics, Lillebaelt Hospital, Vejle, Denmark

${ }^{6}$ Department of Orthopedics and Traumatology, Odense University Hospital, Svendborg, Denmark

${ }^{7}$ Department of Orthopedics, Clinical Sciences Lund, University of Lund, Lund, Sweden

${ }^{8}$ Clinical Epidemiology Research and Training Unit, Boston University School of Medicine, Boston, Massachusetts, USA

Contributors JBT and LSL conceived the study. All authors participated in the study design. JBT, RC and LSL drafted the manuscript. All authors participated in critical scrutinising and revision of the manuscript and approved the final version

Funding This study is supported by grants from The Danish Council for Independent Research | Medical Sciences (\#12-125457) and the Region of Southern Denmark (\#12/6334). Musculoskeletal Statistics Unit, The Parker Institute is supported by grants from the Oak Foundation.

Competing interests None.

Patient consent Obtained.

Provenance and peer review Not commissioned; externally peer reviewed.

Open Access This is an Open Access article distributed in accordance with the Creative Commons Attribution Non Commercial (CC BY-NC 3.0) license, which permits others to distribute, remix, adapt, build upon this work noncommercially, and license their derivative works on different terms, provided the original work is properly cited and the use is non-commercial. See: http:// creativecommons.org/licenses/by-nc/3.0/

\section{REFERENCES}

1. Cullen KA, Hall MJ, Golosinskiy A. Ambulatory surgery in the United States, 2006. National Health Statistics Reports No. 11 revised. Hyattsville, MD: National Center for Health Statistics; 2009.

2. Englund M, Roemer FW, Hayashi D, et al. Meniscus pathology, osteoarthritis and the treatment controversy. Nat Rev Rheumatol 2012;8:412-19.

3. Matthews P, St-Pierre DM. Recovery of muscle strength following arthroscopic meniscectomy. J Orthop Sports Phys Ther 1996;23:18-26.

4. Moffet H, Richards CL, Malouin F, et al. Impact of knee extensor strength deficits on stair ascent performance in patients after medial meniscectomy. Scand J Rehabil Med 1993;25:63-71.

5. St-Pierre DM, Laforest $\mathrm{S}$, Paradis $\mathrm{S}$, et al. Isokinetic rehabilitation after arthroscopic meniscectomy. Eur J Appl Physiol Occup Physiol 1992;64:437-43.

6. Ericsson YB, Roos EM, Dahlberg L. Muscle strength, functional performance, and self-reported outcomes four years after arthroscopic partial meniscectomy in middle-aged patients. Arthritis Rheum 2006:55:946-52.

7. Roos EM, Roos HP, Ryd L, et al. Substantial disability 3 months after arthroscopic partial meniscectomy: a prospective study of patient-relevant outcomes. Arthroscopy 2000;16:619-26.

8. Thorlund JB, Aagaard P, Roos EM. Thigh muscle strength, functional capacity, and self-reported function in patients at high risk of knee osteoarthritis compared with controls. Arthritis Care Res (Hoboken) 2010;62:1244-51.
9. Englund M, Guermazi A, Roemer FW, et al. Meniscal pathology on $\mathrm{MRI}$ increases the risk for both incident and enlarging subchondral bone marrow lesions of the knee: the MOST Study. Ann Rheum Dis 2010;69:1796-802.

10. Moseley JB, O'Malley K, Petersen NJ, et al. A controlled trial of arthroscopic surgery for osteoarthritis of the knee. $N$ Engl $J$ Med 2002;347:81-8.

11. Buchbinder $\mathrm{R}$, Osborne $\mathrm{RH}$, Ebeling $\mathrm{PR}$, et al. A randomized trial of vertebroplasty for painful osteoporotic vertebral fractures. $N$ Engl J Med 2009;361:557-68.

12. Camanho GL, Hernandez AJ, Bitar AC, et al. Results of meniscectomy for treatment of isolated meniscal injuries: correlation between results and etiology of injury. Clinics 2006;61:133-8.

13. Fabricant PD, Rosenberger PH, Jokl $\mathrm{P}$, et al. Predictors of short-term recovery differ from those of long-term outcome after arthroscopic partial meniscectomy. Arthroscopy 2008;24:769-78.

14. Paxton ES, Stock MV, Brophy RH. Meniscal repair versus partial meniscectomy: a systematic review comparing reoperation rates and clinical outcomes. Arthroscopy 2011;27:1275-88.

15. Meredith DS, Losina E, Mahomed NN, et al. Factors predicting functional and radiographic outcomes after arthroscopic partial meniscectomy: a review of the literature. Arthroscopy 2005;21:211-23.

16. Poehling GG, Ruch DS, Chabon SJ. The landscape of meniscal injuries. Clin Sports Med 1990;9:539-49.

17. Englund $M$, Guermazi $A$, Gale $D$, et al. Incidental meniscal findings on knee MRI in middle-aged and elderly persons. $N$ Engl J Med 2008;359:1108-15.

18. Sun Y, Mauerhan DR. Meniscal calcification, pathogenesis and implications. Curr Opin Rheumatol 2012;24:152-7.

19. Englund M, Felson DT, Guermazi A, et al. Risk factors for medial meniscal pathology on knee MRI in older US adults: a multicentre prospective cohort study. Ann Rheum Dis 2011;70:1733-9.

20. Rytter S, Jensen LK, Bonde JP, et al. Occupational kneeling and meniscal tears: a magnetic resonance imaging study in floor layers. J Rheumatol 2009;36:1512-19.

21. Englund M. The role of the meniscus in osteoarthritis genesis. Rheum Dis Clin North Am 2008;34:573-79.

22. Lohmander LS, Englund PM, Dahl LL, et al. The long-term consequence of anterior cruciate ligament and meniscus injuries: osteoarthritis. Am J Sports Med 2007;35:1756-69.

23. Englund M, Guermazi A, Roemer FW, et al. Meniscal tear in knees without surgery and the development of radiographic osteoarthritis among middle-aged and elderly persons: the Multicenter Osteoarthritis Study. Arthritis Rheum 2009;60:831-9.

24. Kirkley A, Birmingham TB, Litchfield RB, et al. A randomized trial of arthroscopic surgery for osteoarthritis of the knee. N Engl J Med 2008;359:1097-107.

25. Herrlin S, Hallander M, Wange $\mathrm{P}$, et al. Arthroscopic or conservative treatment of degenerative medial meniscal tears: a prospective randomised trial. Knee Surg Sports Traumatol Arthrosc 2007;15:393-401.

26. Katz JN, Brophy $\mathrm{RH}$, Chaisson $\mathrm{CE}$, et al. Surgery versus physical therapy for a meniscal tear and osteoarthritis. $N$ Engl J Med 2013;368:1675-84.

27. Herrlin SV, Wange PO, Lapidus G, et al. Is arthroscopic surgery beneficial in treating non-traumatic, degenerative medial meniscal tears? A five year follow-up. Knee Surg Sports Traumatol Arthrosc 2013;21:358-64.

28. Englund M, Roos EM, Roos HP et al. Patient-relevant outcomes fourteen years after meniscectomy: influence of type of meniscal tear and size of resection. Rheumatology (Oxford) 2001;40:631-9.

29. Stein T, Mehling AP, Welsch F, et al. Long-term outcome after arthroscopic meniscal repair versus arthroscopic partial meniscectomy for traumatic meniscal tears. Am J Sports Med 2010;38:1542-8.

30. Peat G, McCarney R, Croft P. Knee pain and osteoarthritis in older adults: a review of community burden and current use of primary health care. Ann Rheum Dis 2001:60:91-7.

31. Sangha $O$, Stucki $G$, Liang $M H$, et al. The Self-Administered Comorbidity Questionnaire: a new method to assess comorbidity for clinical and health services research. Arthritis Rheum 2003;49:156-63.

32. Saltin B, Grimby G. Physiological analysis of middle-aged and old former athletes. Comparison with still active athletes of the same ages. Circulation 1968;38:1104-15

33. Ingham SL, Moody A, Abhishek A, et al. Development and validation of self-reported line drawings for assessment of knee malalignment and foot rotation: a cross-sectional comparative study. BMC Med Res Methodol 2010;10:57.

34. Anderson AF, Irrgang JJ, Dunn W, et al. Interobserver reliability of the International Society of Arthroscopy, Knee Surgery and 
Orthopaedic Sports Medicine (ISAKOS) classification of meniscal tears. Am J Sports Med 2011;39:926-32.

35. Roos EM, Roos HP, Ekdahl C, et al. Knee injury and Osteoarthritis Outcome Score (KOOS) - validation of a Swedish version. Scand J Med Sci Sports 1998;8:439-48.

36. Roos EM, Roos HP, Lohmander LS, et al. Knee Injury and Osteoarthritis Outcome Score (KOOS)-development of a self-administered outcome measure. J Orthop Sports Phys Ther 1998;28:88-96.

37. Roos EM, Lohmander LS. The Knee injury and Osteoarthritis Outcome Score (KOOS): from joint injury to osteoarthritis. Health Qual Life Outcomes 2003;1:64.

38. Tubach $\mathrm{F}$, Ravaud $\mathrm{P}, \mathrm{Baron} \mathrm{G}$, et al. Evaluation of clinically relevant states in patient reported outcomes in knee and hip osteoarthritis: the patient acceptable symptom state. Ann Rheum Dis 2005;64:34-7.

39. Bjorner JB, Damsgaard MT, Watt T, et al. Tests of data quality, scaling assumptions, and reliability of the Danish SF-36. J Clin Epidemiol 1998;51:1001-11.

40. Bjorner JB, Thunedborg K, Kristensen TS, et al. The Danish SF-36 Health Survey: translation and preliminary validity studies. J Clin Epidemiol 1998:51:991-9.

41. Nilsdotter AK, Toksvig-Larsen S, Roos EM. Knee arthroplasty: are patients' expectations fulfilled? A prospective study of pain and function in 102 patients with 5-year follow-up. Acta Orthop 2009;80:55-61.

42. Wells G, Beaton D, Shea B, et al. Minimal clinically important differences: review of methods. J Rheumatol 2001;28:406-12.

43. Paulsen A, Overgaard S, Lauritsen JM. Quality of data entry using single entry, double entry and automated forms processing-an example based on a study of patient-reported outcomes. PLOS ONE 2012;7:e35087.

44. Vandenbroucke JP, von Elm E, Altman DG, et al. Strengthening the Reporting of Observational Studies in Epidemiology (STROBE): explanation and elaboration. Ann Intern Med 2007;147:W163-94.

45. Hare KB, Lohmander LS, Christensen R, et al. Arthroscopic partial meniscectomy in middle-aged patients with mild or no knee osteoarthritis: a protocol for a double-blind, randomized sham-controlled multi-centre trial. BMC Musculoskelet Disord 2013;14:71.

46. Sihvonen R, Paavola M, Malmivaara A, et al. Finnish Degenerative Meniscal Lesion Study (FIDELITY): a protocol for a randomised, placebo surgery controlled trial on the efficacy of arthroscopic partial meniscectomy for patients with degenerative meniscus injury with a novel 'RCT within-a-cohort' study design. BMJ Open 2013;3:pii: e002510.

47. Drosos GI, Pozo JL. The causes and mechanisms of meniscal injuries in the sporting and non-sporting environment in an unselected population. Knee 2004;11:143-9. 